\title{
Oxygen Incorporation in ZnTeO Alloys via Molecular Beam Epitaxy
}

\author{
LI QIN ZHOU, ${ }^{1}$ CHIHYU CHEN ${ }^{2}$ HONGFEI JIA, ${ }^{1,4}$ CHEN LING, ${ }^{1}$ \\ DEBASISH BANERJEE ${ }^{1}$ JAMIE D. PHILLIPS, ${ }^{2,5}$ \\ and YONGQIANG WANG ${ }^{3}$
}

\begin{abstract}
1.-Materials Research Department, Toyota Research Institute of North America, Ann Arbor, MI 48105, USA. 2.-Department of Electrical Engineering and Computer Science, University of Michigan, Ann Arbor, MI 48109, USA. 3.-Ion Beam Materials Laboratory, Materials Science and Technology Division, Los Alamos National Laboratory, Los Alamos, NM 87545, USA. 4.-e-mail: hongfei.jia@tema.toyota.com. 5.—e-mail: jphilli@umich.edu
\end{abstract}

Highly mismatched $\mathrm{ZnTeO}$ alloys were grown by molecular beam epitaxy under various oxygen partial pressures $\left(10^{-7}\right.$ Torr, $10^{-6}$ Torr, and $10^{-5}$ Torr $)$. Despite this large variation in oxygen partial pressure, there was almost no change in the concentration of incorporated oxygen. However, increasing the oxygen partial pressure significantly enhanced the photoluminescence of the oxygen-related transition at $1.9 \mathrm{eV}$. The evolution of lattice constants, as determined by high-resolution x-ray diffraction, appeared to follow Vegard's law for ideal substitutional alloys for $\mathrm{ZnTeO}$ films prepared at $10^{-7}$ Torr and $10^{-6}$ Torr, while a pronounced deviation occurred at $10^{-5}$ Torr. Channeling nuclear reaction analysis further revealed the complexity of oxygen incorporation, as no significant shadowing was observed from the epitaxial films. The evidence suggests that the oxygen location in $\mathrm{ZnTeO}$ is more complicated than $\mathrm{O}-\mathrm{Te}$ substitutional defects in a homogeneous solid solution, and that the configuration of the oxygen dopant might play an important role in the band structure and optical properties of the alloys.

Key words: ZnTeO, highly mismatched alloy, oxygen defect, molecular beam epitaxy

\section{INTRODUCTION}

Formation of intermediate electron states within the forbidden bandgap of a semiconductor can provide a parallel path for excitation of charge carriers to the conduction band by absorbing photons with energy lower than the intrinsic bandgap. Such materials, namely intermediate-band (IB) semiconductors, are of great interest for the development of low-cost solar cells as an alternative to tandem junctions for high solar energy conversion efficiency. ${ }^{1-3}$ Early demonstrations of the IB concept mainly involved addition of InAs quantum dot (QD) arrays to GaAs, where the IB originated from the energy levels of the confined states in the QDs.,

(Received August 1, 2013; accepted December 19, 2013;

published online January 14, 2014)
Although basic IB principles have been verified, small subbandgap photocurrents and loss of output voltage were found to be quite common and difficult to overcome under this configuration of solar cells. ${ }^{6}$ More recently, intermediate-band solar cells (IBSCs) using bulk materials have also been demonstrated, especially those based on highly mismatched alloys (HMAs) ${ }^{7-9}$ in which a small fraction of the host atoms are replaced by an isoelectronic element of very different electronegativity and/or size. IB formation has been confirmed in both nitrogen-doped III-V and oxygen-doped II-VI HMAs. ${ }^{2,3,7-11}$

Despite the bold predictions of high solar energy conversion efficiency for IB materials, demonstration of improved performance over standard semiconductors has not been achieved yet. The success of IB solar cells will likely rely on not only mastering the technology for materials and device preparation 
but also fundamental research to identify the desired spectrum of electronic states, carrier generation and recombination rates, and carrier transport behaviors. As dopants are the origin of IB formation in HMAs, it is critical to understand how they are incorporated into the host materials, and their impact on the band structure and other material properties. Substantial efforts have been made to investigate the location of nitrogen dopant in GaAsN HMAs. ${ }^{12-17}$ In addition to substitutional placement, a considerable fraction of the nitrogen might present as $\mathrm{N}-\mathrm{N}$ or $\mathrm{N}-\mathrm{As}$ split interstitials. ${ }^{12}$ As nitrogen is sensitive to nuclear reaction analysis (NRA), channeling NRA provided direct experimental evidence for the identification of such interstitial nitrogen. ${ }^{13-17}$ Further study also revealed that the decrease of the interstitial $\mathrm{N}$ concentration induced by rapid thermal annealing could result in an increase in carrier concentration and mobility. ${ }^{18}$

Compared with N-doped III-V HMAs, there is much poorer understanding of oxygen incorporation in II-VI alloys. To date, only a few reports on the synthesis and fundamental optical properties of $\mathrm{ZnTeO}$ and $\mathrm{ZnMnTeO}$ have been published. ${ }^{7,8,10,11}$ We previously synthesized epitaxial $\mathrm{ZnTeO}$ films via both pulsed laser deposition (PLD) and molecular beam epitaxy (MBE). ${ }^{8}$ The PLD process was found to result largely in the formation of $\mathrm{TeO}_{x}$, while oxygen was successfully introduced into the lattice of $\mathrm{ZnTe}$ by MBE. A solar cell device using MBE $\mathrm{ZnTeO}$ showed extended spectral response to subbandgap photons, and also demonstrated a $50 \%$ increase in power conversion efficiency compared with an undoped $\mathrm{ZnTe}$ reference cell. ${ }^{2}$ Although an oxygen-induced IB has been confirmed by multiple research groups, the placement of oxygen dopant in $\mathrm{ZnTe}$ has not been clarified yet. Studies on $\mathrm{ZnTeO}$ thus far have primarily assumed substitutional O-Te incorporation to be the dominant defect. However, it can be expected that oxygen incorporation at alternative lattice locations could strongly influence the optoelectronic properties. Our simulations on $\mathrm{ZnTeO}$ using density functional theory (DFT) predicted a strong dependence of the band structure on oxygen configurations. ${ }^{19}$ In this work, epitaxial $\mathrm{ZnTeO}$ films synthesized by MBE under various oxygen partial pressures were characterized by photoluminescence (PL), x-ray photoelectron spectroscopy (XPS), high-resolution x-ray diffraction (XRD), and channeling nuclear reaction analysis (channeling NRA) to study the influence of oxygen incorporation.

\section{EXPERIMENTAL PROCEDURES}

ZnTeO thin films were synthesized on GaAs (001) substrates by MBE following the same procedure as reported previously. ${ }^{8}$ Epitaxial film growth was performed with base pressure of $10^{-9}$ Torr at substrate temperature of $300^{\circ} \mathrm{C}$ using zinc and tellurium solid-source effusion cells and a microwave plasma source to introduce oxygen. Different oxygen partial pressures of $10^{-7}$ Torr, $10^{-6}$ Torr, and $10^{-5}$ Torr were applied during film deposition. Intrinsic ZnTe film without oxygen doping was also prepared as control. XPS analysis was conducted using a PHI 5000 VersaProbe II system to verify the presence of oxygen and associated bonding configurations. High-resolution Te $3 d$ and $\mathrm{O} 1 s$ scans were performed with pass energy of $23.5 \mathrm{eV}$ and scan step of $0.1 \mathrm{eV}$. To examine the bulk materials, the top surface of the films was removed by in situ Ar sputtering. PL measurements were performed using a HeCd laser operating at $325 \mathrm{~nm}$, monochromator, and closed-cycle helium cryostat. XRD patterns were obtained using a Rigaku SmartLab x-ray diffractometer with $\mathrm{Cu} \mathrm{K}_{\alpha}$ radiation $(\lambda=1.5405 \AA)$. Channeling NRA experiments were conducted at Los Alamos National Laboratory using a $1.05-\mathrm{MeV}$ deuteron $\left(\mathrm{D}^{+}\right)$beam generated by a 3-MV Pelletron
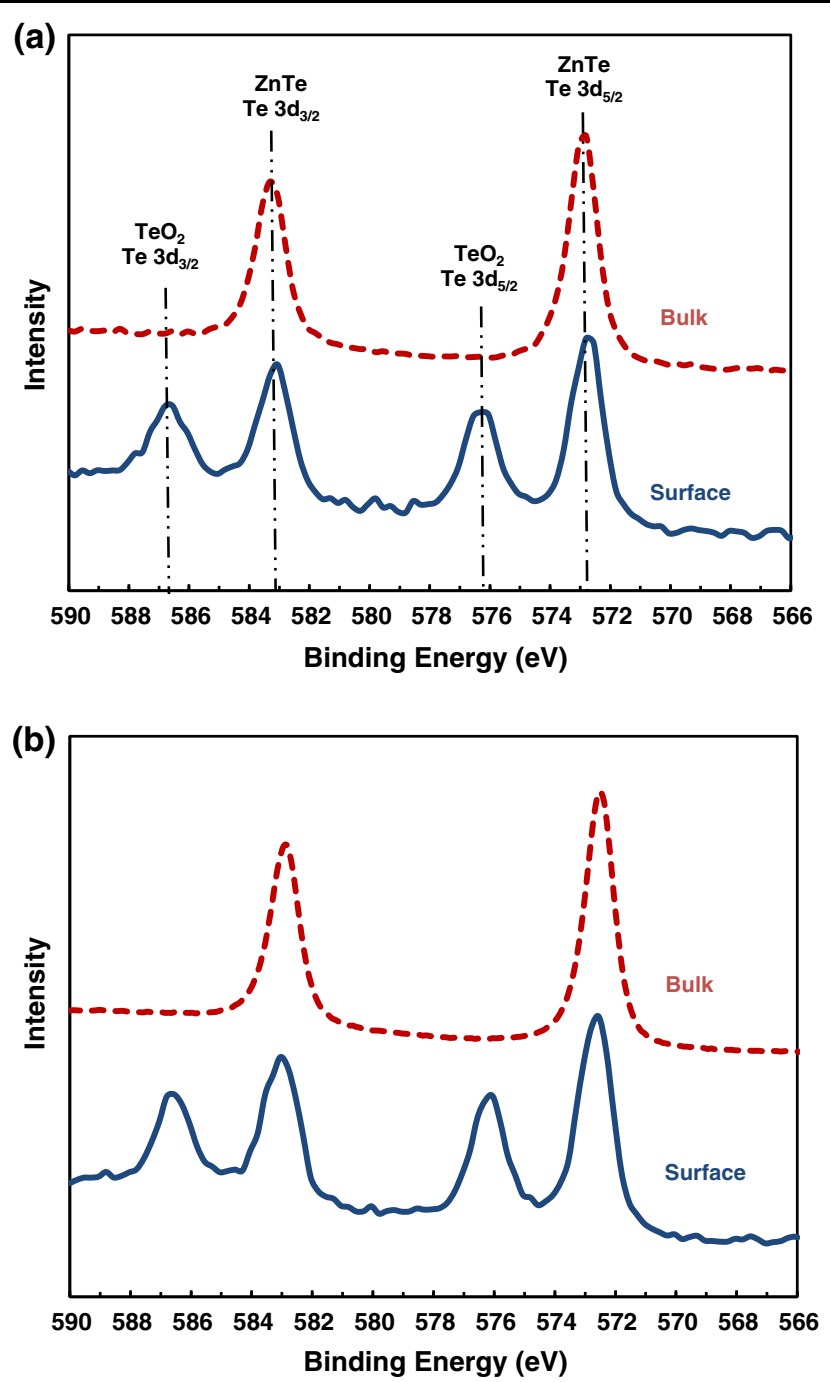

Fig. 1. Te $3 d_{3 / 2}$ and Te $3 d_{5 / 2}$ XPS results from the surface and bulk of (a) intrinsic ZnTe film and (b) ZnTeO film deposited at $10^{-5}$ Torr. Peak intensity is normalized to $\mathrm{Te} 3 d_{5 / 2}$ peak. $\mathrm{TeO}_{2}$ was present only on the surface. $\mathrm{No}_{\mathrm{TeO}}$ was formed during film growth. 


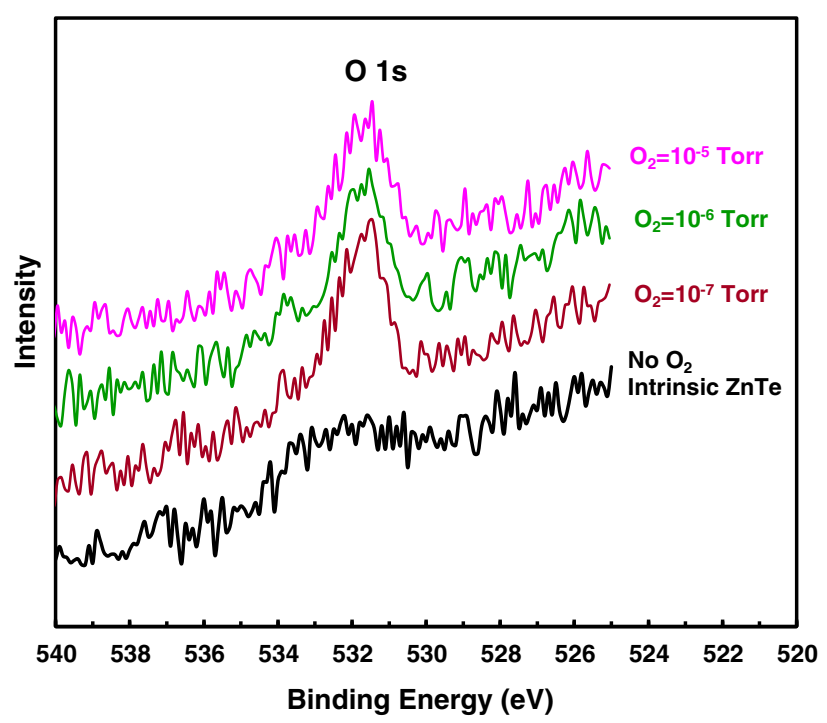

Fig. 2. O $1 s$ XPS results for bulk of intrinsic ZnTe and ZnTeO films grown at $10^{-7}$ Torr, $10^{-6}$ Torr, and $10^{-5}$ Torr. The intensity is normalized to Te $3 d_{5 / 2}$ peak.

tandem ion accelerator. The nuclear reaction $\mathrm{O}^{16}(\mathrm{~d}$, p) $\mathrm{O}^{17}$ was used to detect oxygen by measuring emitted protons with a silicon surface barrier detector placed at $135^{\circ}$ from the beam direction. Kapton foil (18 $\mu \mathrm{m}$ thick) was placed in front of the detector to filter out deuterium particles backscattered from the sample. Another silicon surface barrier detector was placed at $167^{\circ}$ to measure deuterium particles backscattered from the $\mathrm{ZnTe}$ target using Rutherford backscattering spectrometry (RBS). The samples were mounted on a five-axis goniometer with lateral resolution of $1 \mu \mathrm{m}$ and angular resolution of $0.01^{\circ}$.

\section{RESULTS AND DISCUSSION}

Doping oxygen into the lattice of $\mathrm{ZnTe}$ is very challenging due to the highly mismatched electronegativity and size between oxygen and tellurium. Various oxygen partial pressures $\left(10^{-7}\right.$ Torr, $10^{-6}$ Torr, and $10^{-5}$ Torr) were applied during the film growth with the expectation of enhanced oxygen incorporation under higher pressure. To verify the presence of oxygen in the films, XPS analysis was conducted. Figure 1 compares the XPS spectra of tellurium for the intrinsic ZnTe (without oxygen doping) film and the oxygen-doped $\mathrm{ZnTeO}$ sample grown at $10^{-5}$ Torr. $\mathrm{TeO}_{2} 3 / 2$ and $5 / 2$ peaks were observed from the surface for both the intrinsic and oxygen-doped samples, while they were not found from the bulk of the films, indicating that no $\mathrm{TeO}_{2}$ was formed during film growth. The same phenomena were observed for $\mathrm{ZnTeO}$ samples grown under $10^{-6}$ Torr and $10^{-7}$ Torr oxygen partial pressures. The $\mathrm{O} 1 \mathrm{~s}$ profile scans from the bulk of the films are shown in Fig. 2. The spectrum for the intrinsic ZnTe sample is also included in the figure as reference. $\mathrm{O} 1 s$ peaks were clearly observed from

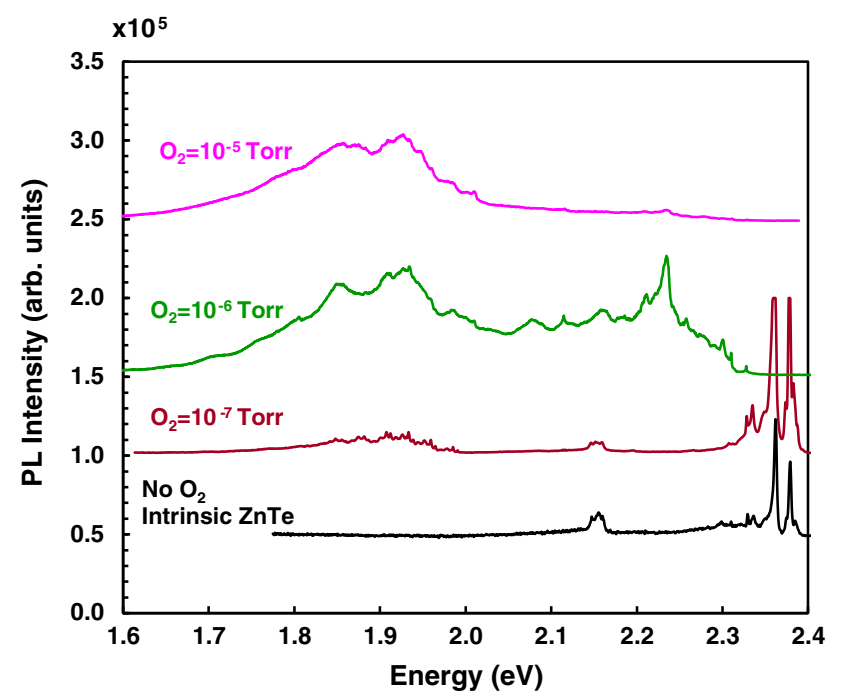

Fig. 3. PL spectra at $20 \mathrm{~K}$ for intrinsic $\mathrm{ZnTe}$ and oxygen-doped $\mathrm{ZnTeO}$ films grown at $10^{-7}$ Torr, $10^{-6}$ Torr, and $10^{-5}$ Torr.

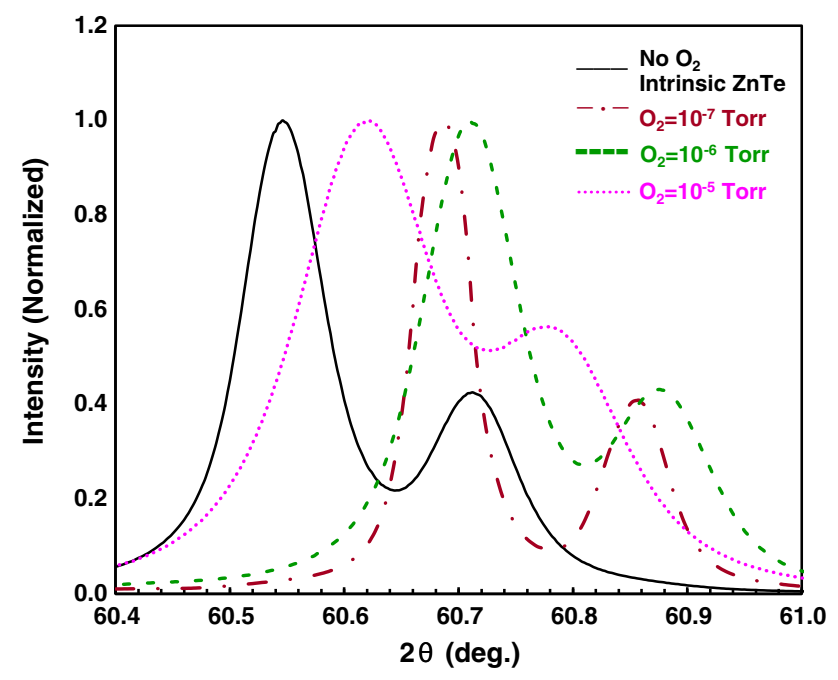

Fig. 4. XRD profiles of (004) plane for intrinsic ZnTe and oxygendoped $\mathrm{ZnTeO}$ grown at $10^{-7}$ Torr, $10^{-6}$ Torr, and $10^{-5}$ Torr.

all of the oxygen-doped $\mathrm{ZnTeO}$ films, while no peak was detected from the ZnTe reference sample. To our surprise, no appreciable difference was observed among the $\mathrm{O} 1 s$ profiles of the samples grown under different oxygen partial pressures, indicating that all of them had a similar oxygen concentration. Independent measurements using NRA confirmed that the $\mathrm{O}$ concentration was approximately 1 at.\% for all the samples grown at different oxygen partial pressures. Secondary-ion mass spectrometry (SIMS) analysis further confirmed that the oxygen concentration lay in this range. These results seem to be different from a report by Tanaka et al. ${ }^{11}$ where a nearly linear increase in $\mathrm{O}$ concentration up to $4.6 \%$ was observed with increase in $\mathrm{O}_{2}$ flow rate. Oxygen incorporation in the MBE process can be affected by 
Table I. Calculated lattice constant and oxygen concentration based on XRD measurements

\begin{tabular}{|c|c|c|c|}
\hline $\begin{array}{l}\text { Oxygen Partial } \\
\text { Pressure (Torr) }\end{array}$ & Measured 2 $\left(^{\circ}\right)$ & $\begin{array}{l}\text { Calculated Lattice } \\
\text { Constant (̊) }\end{array}$ & $\begin{array}{l}\text { O Concentration (at.\%) Cal- } \\
\text { culated from Vegard's Law }\end{array}$ \\
\hline 0 (intrinsic $\mathrm{ZnTe}$ ) & 60.546 & 6.112 & \\
\hline $10^{-7}$ & 60.686 & 6.099 & 0.84 \\
\hline $10^{-6}$ & 60.708 & 6.097 & 0.98 \\
\hline $10^{-5}$ & 60.622 & 6.105 & 0.46 \\
\hline
\end{tabular}

many parameters such as the oxygen plasma conditions, growth temperature, growth rate, Te/Zn flux ratio, etc. ${ }^{8}$ Differences in these conditions may have contributed to the difference between our results and the prior report. ${ }^{11}$ Moreover, ZnTe substrate was used in the prior report ${ }^{11}$ while the present films were grown on GaAs substrate, for which the appreciable lattice mismatch between $\mathrm{ZnTe}$ and GaAs ( 8\%) may have a nonnegligible effect on oxygen incorporation. For instance, Nabetani et al. ${ }^{20}$ reported a maximum $O$ concentration of $0.1 \%$ in $\mathrm{ZnTeO}$ grown by MBE on GaAs, much lower than for films obtained on ZnTe substrate.

Photoluminescence spectra measured at $20 \mathrm{~K}$ are shown in Fig. 3. Undoped ZnTe exhibited a clear band-edge emission at $2.3 \mathrm{eV}$ to $2.4 \mathrm{eV}$ that vanished as the oxygen partial pressure reached above $10^{-6}$ Torr. A similar phenomenon was reported where the band-edge emission disappeared for $\mathrm{ZnTeO}$ with even low $\mathrm{O}$ concentration (oxygen flow rate of $0.0035 \mathrm{ccm}){ }^{20}$ For $\mathrm{ZnTeO}$ samples, a weak and broad peak near $1.9 \mathrm{eV}$ was observed, and its intensity increased with rising oxygen partial pressure. This peak was attributed to oxygen incorporation. Furthermore, the $2.2 \mathrm{eV}$ donoracceptor pair band ${ }^{21}$ was observed in the $10^{-6}$ Torr film but greatly suppressed in the $10^{-7}$ Torr and $10^{-5}$ Torr samples. As the total concentration of $\mathrm{O}$ atoms in the film was nearly independent of the oxygen partial pressure, these characteristic differences observed in the PL measurements must derive from a difference in the oxygen configuration in the lattice structure. To examine this hypothesis, high-resolution XRD patterns were acquired, with the results shown in Fig. 4. All samples demonstrated a dominant $\mathrm{ZnTe}(004)$ reflection, indicating a clear ZnTe (001) orientation on GaAs (001). The introduction of oxygen resulted in a shift of the peaks to higher angles. Assuming complete oxygen substitution for Te and using Vegard's law, the lattice constant is given by

$$
a_{\mathrm{ZnTeO}}=x a_{\mathrm{ZnO}}+(1-x) a_{\mathrm{ZnTe}}
$$

where $a_{\mathrm{ZnO}}$ and $a_{\mathrm{ZnTe}}$ are the lattice constants of zincblende $\mathrm{ZnO}\left(4.600 \AA^{22}\right)$ and $\mathrm{ZnTe}$ [6.112 $\mathrm{A}$, calculated using the (004) peak of the intrinsic ZnTe film]. The lattice constant and oxygen concentration for the different films were calculated, and the results are listed in Table I. The estimated $\mathrm{O}$
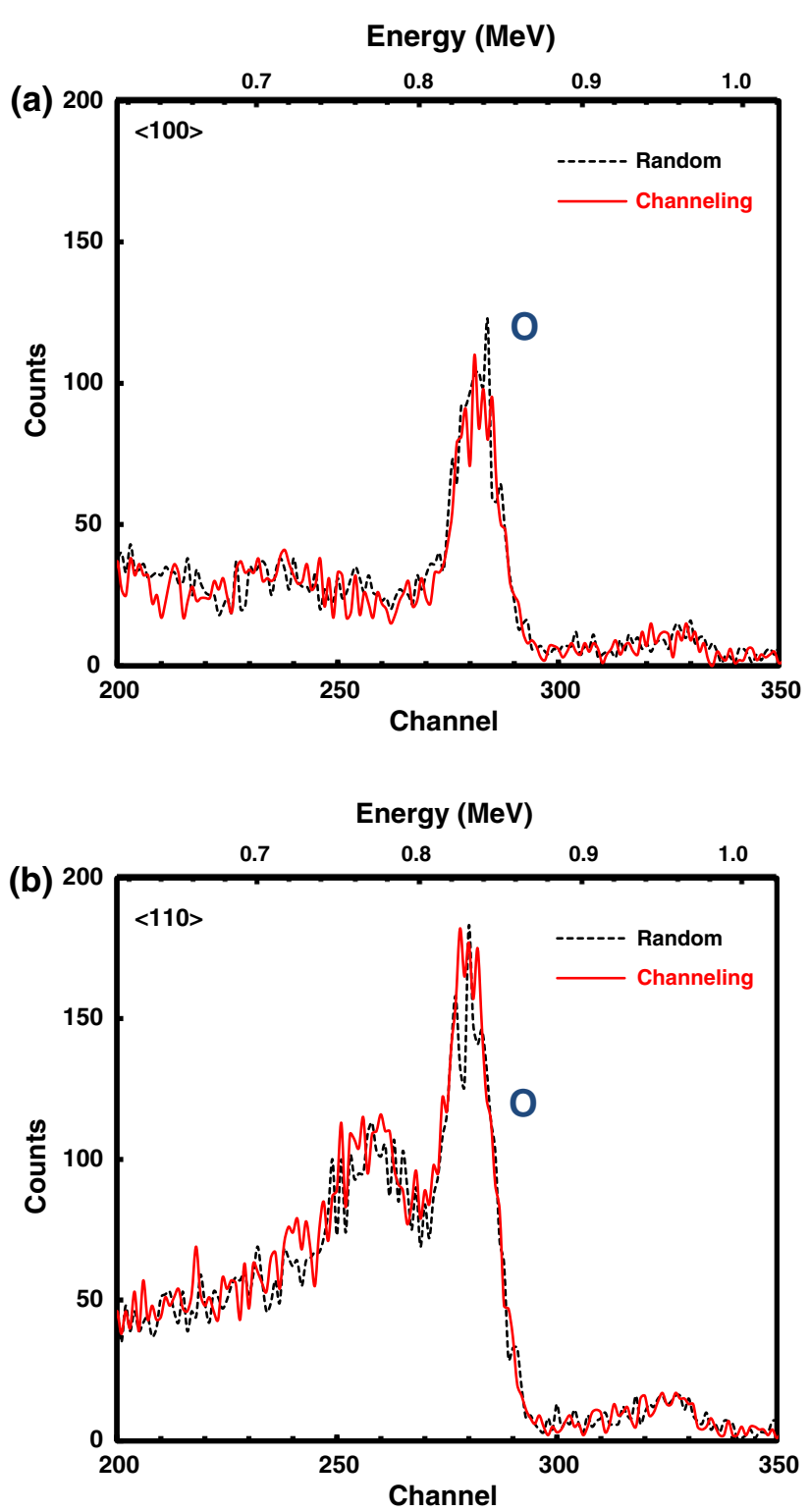

Fig. 5. Channeling and random oxygen spectra of ZnTeO film (grown at $10^{-6}$ Torr) along (a) $<100>$ and (b) $<110>$ orientations.

concentration for the $10^{-7}$ Torr and $10^{-6}$ Torr samples, based on the lattice constant obtained from XRD, is similar to the 1 at.\% value measured by SIMS and NRA. However, there is a pronounced deviation for the $10^{-5}$ Torr sample, which actually suggests a reduced oxygen concentration. Therefore, the 
assumption of O-Te substitution is likely incorrect for higher oxygen partial pressure, where oxygen atoms are likely situated at alternative locations in the lattice and/or form defect complexes. As interstitial $O$ expands while substitutional $O$ shrinks the lattice, the XRD results might reflect the combined effect of substitutional and interstitial O. Although high oxygen partial pressure did not increase the total incorporation of oxygen into $\mathrm{ZnTe}$, it may be increasing the concentration of interstitial oxygen.

The complexity of oxygen locations in $\mathrm{ZnTeO}$ was justified by channeling NRA experiments. In the zincblende structure, ideal substitutional or interstitial oxygen results in different channeling spectra at certain crystallographic directions. ${ }^{23}$ As shown in Fig. 5, no significant changes were observed between the random and channeling oxygen spectra in $<100>$ or $<110>$ directions for the sample grown at $10^{-6}$ Torr. Similar behavior was observed for $10^{-7}$ Torr and $10^{-5}$ Torr films. These data indicate that the oxygen locations in our $\mathrm{ZnTeO}$ films cannot be simply described with the ideal substitutional or interstitial picture. Nonetheless, it is safe to conclude that oxygen indeed occupies various defect sites in $\mathrm{ZnTeO}$. The PL measurements (Fig. 3) and our previous DFT calculations ${ }^{19}$ both suggested that the band structures and optoelectronic properties of $\mathrm{ZnTeO}$ strongly depend on oxygen locations. Identification of the $\mathrm{O}$ status in $\mathrm{ZnTeO}$ is therefore believed to be critical to understand the mechanism of IB formation. Efforts are still being made to further clarify the location of oxygen and how it affects the performance of $\mathrm{ZnTeO}$ as a solar adsorbent.

\section{CONCLUSIONS}

Oxygen incorporation was investigated in highly mismatched $\mathrm{ZnTeO}$ alloys synthesized by MBE. The total oxygen concentration was nearly independent of the oxygen partial pressure, being about 1 at.\% as estimated by SIMS and NRA. However, the placement of the oxygen in the lattice might be different, resulting in different characteristic features in PL and the deviation of the lattice constant of the alloys from Vegard's law. Evidence from channeling NRA suggested that oxygen was not a direct substitutional impurity in the alloy films and may be located at various sites in the crystal lattice. As quantitative detection of oxygen lattice location is not a trivial task, additional efforts are still needed to obtain a clear picture of oxygen incorporation in the alloy films. It can be expected that final clarification of oxygen status would be greatly beneficial to the development of IB materials for solar cell and other practical applications.

\section{ACKNOWLEDGEMENTS}

This work was performed, in part, at the Center for Integrated Nanotechnologies, an Office of Science User Facility operated jointly by Los Alamos and Sandia National Laboratories. Los Alamos National Laboratory, an affirmative action equal opportunity employer, is operated by Los Alamos National Security, LLC, for the National Nuclear Security Administration of the US Department of Energy under Contract DE-AC52-06NA25396.

\section{REFERENCES}

1. A. Luque and A. Marti, Phys. Rev. Lett. 78, 5014 (1997).

2. W. Wang, A. Lin, and J. Phillips, Appl. Phys. Lett. 95, 011103 (2009).

3. T. Tanaka, K.M. Yu, P.R. Stone, J.W. Beeman, O.D. Dubon, L.A. Reichertz, V.M. Kao, M. Nishio, and W. Walukiewicz, J. Appl. Phys. 108, 024502 (2010).

4. A. Marti, E. Antolin, C.R. Stanley, C.D. Farmer, N. Lopez, P. Diaz, E. Canovas, P.G. Linares, and A. Luque, Phys. Rev. Lett. 97, 247701 (2006).

5. R. Oshima, A. Takata, and Y. Okada, Appl. Phys. Lett. 93, 083111 (2008).

6. A. Luque, A. Marti, and C. Stanley, Nat. Photonics 6, 146 (2012).

7. K.M. Yu, W. Walukiewicz, W. Shan, J. Wu, J.W. Beeman, M.A. Scarpulla, O.D. Dubon, and P. Becla, J. Appl. Phys. 95, 6232 (2004).

8. W. Wang, W. Bowen, S. Spanninga, S. Lin, and J. Phillips, J. Electron. Mater. 38, 119 (2009).

9. N. Lopez, L.A. Reichertz, K.M. Yu, K. Campman, and W. Walukiewicz, Phys. Rev. Lett. 106, 028701 (2011).

10. K.M. Yu, W. Walukiewicz, J. Wu, W. Shan, J.W. Beeman, M.A. Scarpulla, O.D. Dubon, and P. Becla, Phys. Rev. Lett. 91, 246403 (2003).

11. T. Tanaka, S. Kusaba, T. Mochinaga, K. Saito, Q. Guo, M. Nishio, K.M. Yu, and W. Walukiewicz, Appl. Phys. Lett. 100, 011905 (2012).

12. S.B. Zhang and S.H. Wei, Phys. Rev. Lett. 86, 1789 (2001).

13. J.N. Beaudry, R.A. Masut, P. Desjardins, R. Wei, M. Chicoine, G. Bentoumi, R. Leonelli, F. Schiettekatte, and S. Guillon, J. Vac. Sci. Technol. A 22, 771 (2004).

14. M. Reason, H.A. Mckay, W. Ye, S. Hanson, R.S. Goldman, and V. Rotberg, Appl. Phys. Lett. 85, 1692 (2004).

15. T. Ahlgren, E. Vainonen-Ahlgren, J. Likonen, W. Li, and M. Pessa, Appl. Phys. Lett. 80, 2314 (2002).

16. S.G. Spruytte, C.W. Coldren, J.S. Harris, W. Wampler, P. Krispin, K. Ploog, and M.C. Larson, J. Appl. Phys. 89, 4401 (2001).

17. H. Kobayashi, K. Kimura, F. Nishiyama, S. Miwa, and T. Yao, J. Cryst. Growth 184/185, 475 (1998).

18. Y. Jin, R.M. Jock, H. Cheng, Y. He, A.M. Mintarov, Y. Wang, C. Kurdak, J.L. Merz, and R.S. Goldman, Appl. Phys. Lett. 95, 062109 (2009).

19. C. Ling, L.Q. Zhou, D. Banerjee, and H. Jia, J. Alloys Compd. 583, 289 (2014).

20. Y. Nabetani, T. Okuno, K. Aoki, T. Kato, T. Matsumoto, and T. Hirai, Phys. Status Solidi C 3, 1078 (2006).

21. M. Ekawa, Y. Kawakami, T. Taguchi, and A. Hiraki, J. Cryst. Growth 93, 667 (1988).

22. J.E. Jaffe and A.C. Hess, Phys. Rev. B 48, 7903 (1993).

23. Y. Wang and M. Nastasi, Handbook of Modern Ion Beam Materials Analysis, vol. 2, 2nd ed. (Pittsburgh: Materials Research Society, 1995), p. 324. 